\title{
Electron pitch angle variations recorded at the high magnetic latitude boundary layer by the NUADU instrument on the TC-2 spacecraft
}

\author{
L. Lu ${ }^{1}$, S. McKenna-Lawlor ${ }^{2}$, S. Barabash ${ }^{3}$, Z. X. Liu ${ }^{1}$, J. Balaz ${ }^{2}$, K. Brinkfeldt ${ }^{3}$, I. Strharsky ${ }^{2}$, C. Shen ${ }^{1}$, J. K. Shi ${ }^{1}$, \\ J. B. Cao ${ }^{1}$, S. Y. Fu ${ }^{4}$, H. Gunell ${ }^{2,3}$, K. Kudela ${ }^{5}$, E. C. Roelof $^{6}$, P. C. Brandt ${ }^{6}$, I. Dandouras ${ }^{7}$, T. L. Zhang ${ }^{8}$, C. Carr ${ }^{9}$, \\ and A. Fazakerley ${ }^{10}$ \\ ${ }^{1}$ Centre for Space Science and Applied Research of the Chinese Academy of Sciences, Beijing, China \\ ${ }^{2}$ Space Technology Ireland, National University of Ireland, Maynooth, Co. Kildare, Ireland \\ ${ }^{3}$ Swedish Institute of Space Physics, Kiruna, Sweden \\ ${ }^{4}$ Beijing University, Beijing, China \\ ${ }^{5}$ Institute of Experimental Physics, Kosice, Slovakia \\ ${ }^{6}$ Applied Physics Laboratory, Johns Hopkins University, Maryland, USA \\ ${ }^{7}$ Centre d' Etude Spatialedes Rayonnements Toulouse, France \\ ${ }^{8}$ Space Research Institute, Austrian Academy of Sciences, Graz, Austria \\ ${ }^{9}$ Imperial College of Science, London, UK \\ ${ }^{10}$ MSSL, UCL, Holmbury St. Mary, UK
}

Received: 14 February 2005 - Revised: 4 August 2005 - Accepted: 13 September 2005 - Published: 8 November 2005

Part of Special Issue "Double Star - First Results"

\begin{abstract}
The NUADU (NeUtral Atom Detector Unit) experiment aboard TC-2 recorded, with high temporal and spatial resolution, $4 \pi$ solid angle images of electrons $(\sim 50-$ $125 \mathrm{keV})$ spiraling around geomagnetic field lines at high northern magnetic latitudes ( $\mathrm{L}>10)$, during its in-orbit commissioning phase (September 2004). The ambient magnetic field, as well as electrons in other energy ranges, were simultaneously measured by the TC-2 magnetometer (FGM), the plasma electron and current experiment (PEACE), the low energy ion detector (LEID) and the high energy electron detector (HEED). The NUADU data showed that up-flowing electron beams could form "ring-like" and "dumbbell-type" pitch angle distributions (PADs) in the region sampled. Changes in these pitch angle distributions due to transient magnetic variations are suggested to have been associated with electron acceleration along the geomagnetic field lines. A nested magnetic bottle configuration that formed due to the propagation towards the Earth of a magnetic pulse, is proposed to have been associated with this process.
\end{abstract}

Keywords. Interplanetary physics (Energetic particles) Magnetospheric physics (Storms and substorms; Plasma sheet)

Correspondence to: $\mathrm{L} . \mathrm{Lu}$

(luli@center.cssar.ac.cn)

\section{Introduction}

The Chinese Space Agency launched the second of a pair of spacecraft (TC-2), forming the Double Star Mission, into a polar orbit on 25 July 2004 . TC-2 carries a payload designed to investigate various features of geo-space, both on a stand-alone basis and in co-operation with the CLUSTER mission already launched by the European Space Agency. During its on-orbit commissioning in September 2004, the NUADU instrument imaged the spherical pitch angle distributions (PADs) of ambient electrons in the $\mathrm{keV}$ range at high temporal and spatial resolution, at high northern latitudes $(\mathrm{L}>10)$. This provided an opportunity to investigate, in detail, the formation and evolution of these PADs.

The measurement and investigation of pitch angle distributions provide insight into the physical mechanisms pertaining to energetic plasmas. Usually a PAD is observed to evolve from being nearly perpendicular (pitch angles $\sim 90^{\circ}$ ) to being nearly field-aligned (pancake-type). At the High Magnetic Latitude Boundary Layer (HMLBL), especially during episodes of geomagnetic activity, the distributions may rather be of the dumbbell-type (Asnes et al., 2005). In the spherical view, single ring-like PADs are observed which are referred to as "smoke rings" by Williams (1981). The electron beam concerned is commonly called the "plasma source" of those abnormal PADs, which conserve the first adiabatic invariant during their evolution. Several authors analysed the mechanisms of transport and loss 

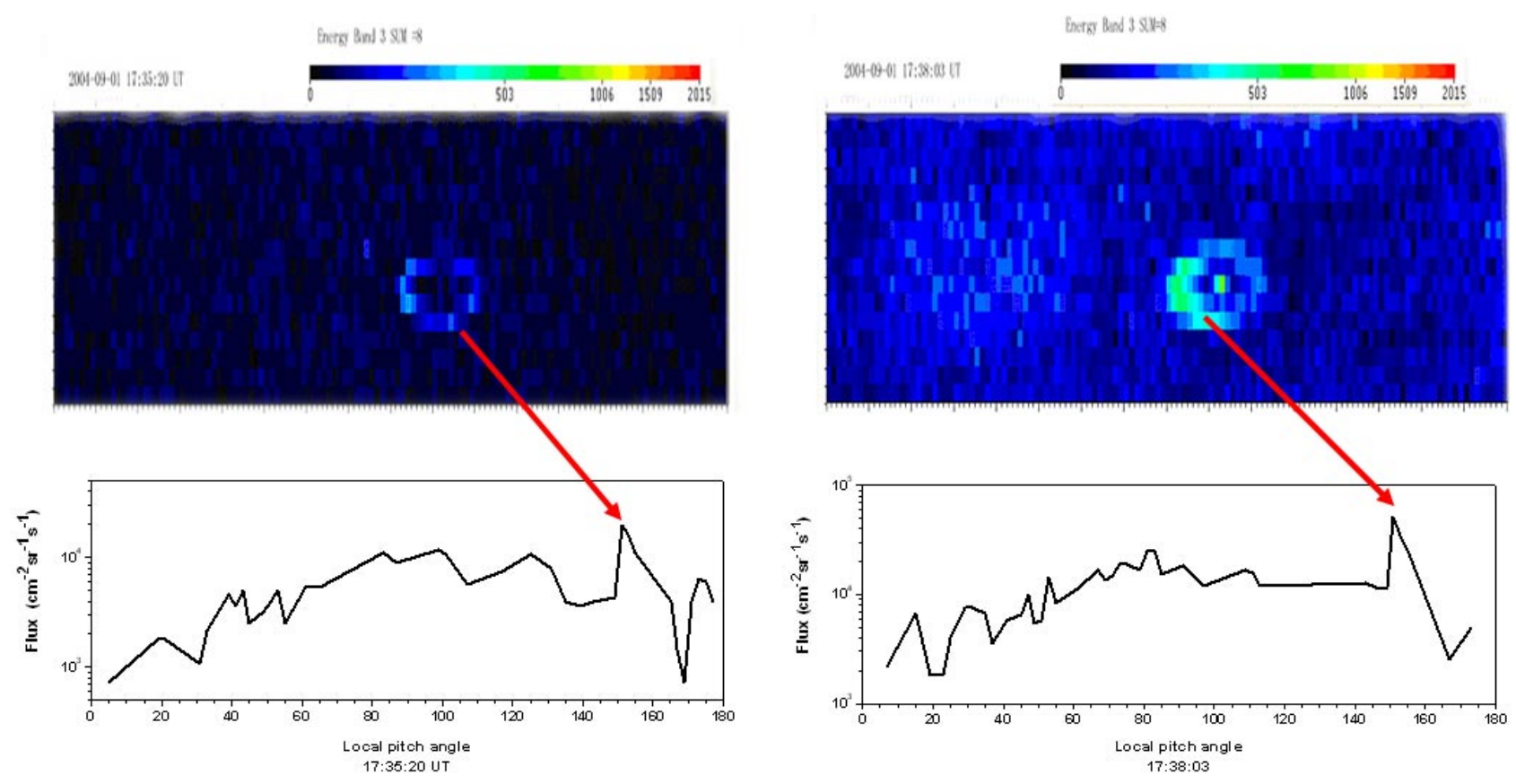

Fig. 1. Top: examples of ring-like particle configurations. Each abscissa covers 128 azimuths, with $2.8125^{\circ}$ to each pixel, and each ordinate covers 16 elevations, with $11.25^{\circ}$ to each pixel. The colour scales at the top indicate the number of particle counts in each pixel. Bottom panel: shows corresponding PAD plots. Red arrows point to the flux peaks formed at the locations of the rings.

Table 1. Energy channels of NUADU.

\begin{tabular}{ccccccc}
\hline Energy Channels & Electron [keV] & $\mathrm{H}[\mathrm{keV}]$ & $\mathrm{O}[\mathrm{keV}]$ & $\Delta \mathrm{E}_{e}[\mathrm{keV}]$ & $\Delta \mathrm{E}_{H}[\mathrm{keV}]$ & $\Delta \mathrm{E}_{O}[\mathrm{keV}]$ \\
\hline E1: TH1-TH2 & $14.26-18.4$ & $45.1-50$ & $*-138$ & 4.1 & 4.9 & - \\
E2: TH2-TH3 & $18.4-49.2$ & $50-81$ & $138-185$ & 30.8 & 31 & 47 \\
E3: TH3-TH4 & $49.2-125.2$ & $81-158$ & $185-300$ & 76 & 77 & 115 \\
\hline
\end{tabular}

of the electrons concerned (e.g. Friedel et al., 2002; Green, 2004). Large-scale (proposed by Fujimoto et al., 1990), and small-scale (proposed by Boscher et al., 2000, and by Liu et al., 1999), re-circulation in the magnetosphere, involving radial diffusion and pitch angle scatting, were each suggested by these authors to result in electron acceleration.

The present study focuses on PADs observed at the HMLBL so as to investigate (a) the correlation between abnormal PADs and the associated behaviour of ambient magnetic fields, and (b) to compare the behaviour of electrons in the $\mathrm{keV}$ range moving along the geo-magnetic field lines observed by NUADU, and local electrons simultaneously recorded at other energies by various instruments of the TC- 2 payload.

Section 2 presents a brief introduction to the NUADU instrument. Sections 3 and 4 describe two instances in which the observed PADs changed in temporal association with changes in the ambient magnetic field. In Sect. 5, these abnormal PADs are discussed and final conclusions presented.

\section{Instrumentation}

The NUADU instrument on TC- 2 features 16 solid-state detectors, each with a rectangular active area of $46.5 \times 10 \mathrm{~mm}^{2}$ and each with an equal field of view $\left(11.5^{\circ}-2.5^{\circ} \mathrm{fwhm}\right)$ distributed regularly over an $180^{\circ}$ angle in the elevation plane. Spacecraft spin (4s) allows the azimuthal plane to be divided into 128 equal sectors through counting pulses provided by the spacecraft, and NUADU can provide a full $4 \pi$ image of the ambient particle population on the completion of each spacecraft spin (4s). It is possible to integrate $\mathrm{N}$ spins onboard within the range $\mathrm{N}=2-32$ to achieve better statistics. For details concerning the instrument, see McKenna-Lawlor et al., 2004.

NUADU can record both neutral and charged particles. In Table 1, the energy channels of the instrument with respect to both neutral atoms and electrons are presented. NUADU was commissioned in space in the period August-September 2004. Due to its high geometric factor $\left(\mathrm{G}=1.3 \times 10^{-3} \mathrm{sr} \mathrm{cm}^{2}\right)$, NUADU saturates in the radiation belts but can record particles at high northern latitudes $(\mathrm{L} \sim 10)$. The viewing 

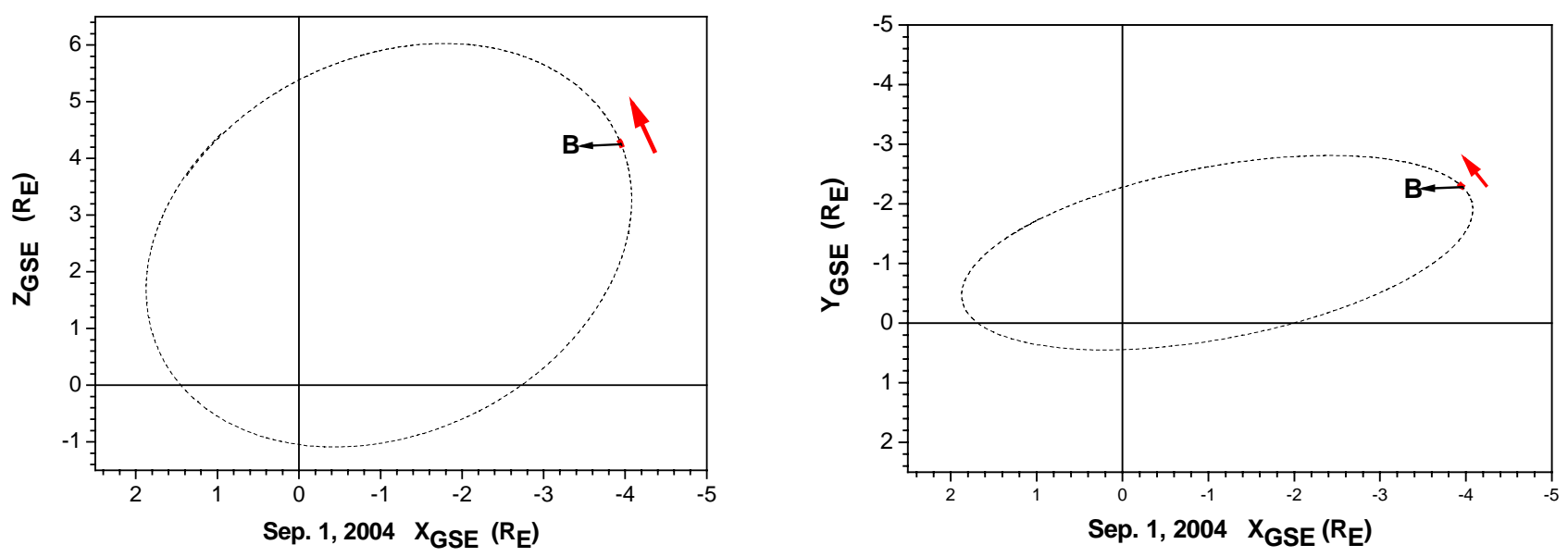

Fig. 2. The location of TC-2 in the X-Y plane (right), and in the $X-Z$ plane (left) in the GSE system. Black arrows indicate the magnetic field direction and red arrows the direction of motion of the spacecraft at the time of the PADs shown in Fig. 1.

capability of the instrument allows the pitch angles of particles spiraling at high magnetic latitudes in the geo-magnetic field to be imaged.

The experiments FGM, PEACE, LEID, and HEED aboard TC-2 (individually described in this issue) simultaneously measured ambient magnetic fields and electrons in several different energy ranges and these data were used (see below) in studying two kinds of abnormal PAD.

\section{Ring-like PADs}

On 1 September 2004 at 17:35:20 UT, and again at 17:38:03 UT, two ring-like particle distributions were imaged in electrons by NUADU in Energy Channel 3 above the radiation belt in the HMLBL (see Fig. 1, top panel). It is noted that a bright point in the centre of the ring distribution shown in the frame, on the right-hand side, is due to cross talk. The bright points at the left side of each frame represent, on the other hand, contributions to the normal PAD background.

Each frame in Fig. 1 was formed by integrating over 8 spacecraft spins. The accumulation time for each pixel was $0.25 \mathrm{~s}$. The PAD curves (Fig. 1, bottom panel) show that, in each case, a particle beam was superposed on the normal PAD background, and that the local pitch angle of the beam was $\sim 152^{\circ}$. Energetic beams in a spherical (smoke ring) distribution were recorded on ISEE 1 at about $-20 \mathrm{R}_{E}$ in the magnetotail (Williams, 1981). However, the strong intensity gradients at the edge of the plasma sheet, and limited temporal-spatial resolution (36-s integration interval for a 192-pixel spherical view frame), resulted in producing obvious azimuthal asymmetries in the associated magnetic field line. Each image in Fig. 1 required 32-s accumulation. However, eight full frames were integrated together, so that the rings remained symmetrical about the magnetic field line.

TC-2 was located at the dawn nightside when at the HMLBL (Fig. 2). The ambient magnetic field was recorded

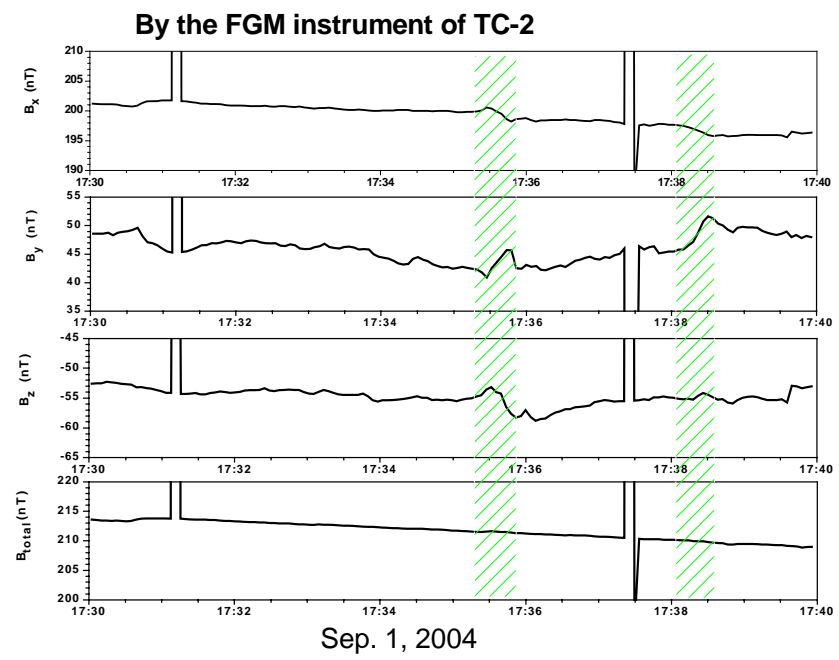

Fig. 3. Magnetic field records recorded by FGM on 1 September 2004. These are, from top to bottom, the X-component $\left(B_{X}\right)$, Ycomponent $\left(B_{Y}\right)$, Z-component $\left(B_{Z}\right)$, and total magnetic field intensity $\left(B_{T}\right)$. The times of the ring particle observations shown in Fig. 1 are coloured in green.

by FGM. The time intervals of the frames under study (shown in Fig. 1) are shaded green on the magnetic field plot presented in Fig. 3. Each magnetic component displayed a transient shift, approximately $5 \mathrm{nT}$, during the individual accumulation periods. This implies that the magnetic flux tube suffered a distortion at these times. It is noted that, some minutes before the formation of the ring-like structures, multiple magnetic spikes (nT) were recorded by FGM which may have been related to the events considered. These spikes presently require further investigation.

Due to the absence of other energetic particle detectors onboard TC-2, we can only consult the low-energy particle observations made by PEACE $(1 \mathrm{eV}-1 \mathrm{keV})$ and LEID $(50 \mathrm{eV}-$ $25 \mathrm{keV}$ ), and the relatively "high-energy" data from HEED $(0.2-0.4 \mathrm{MeV})$. 

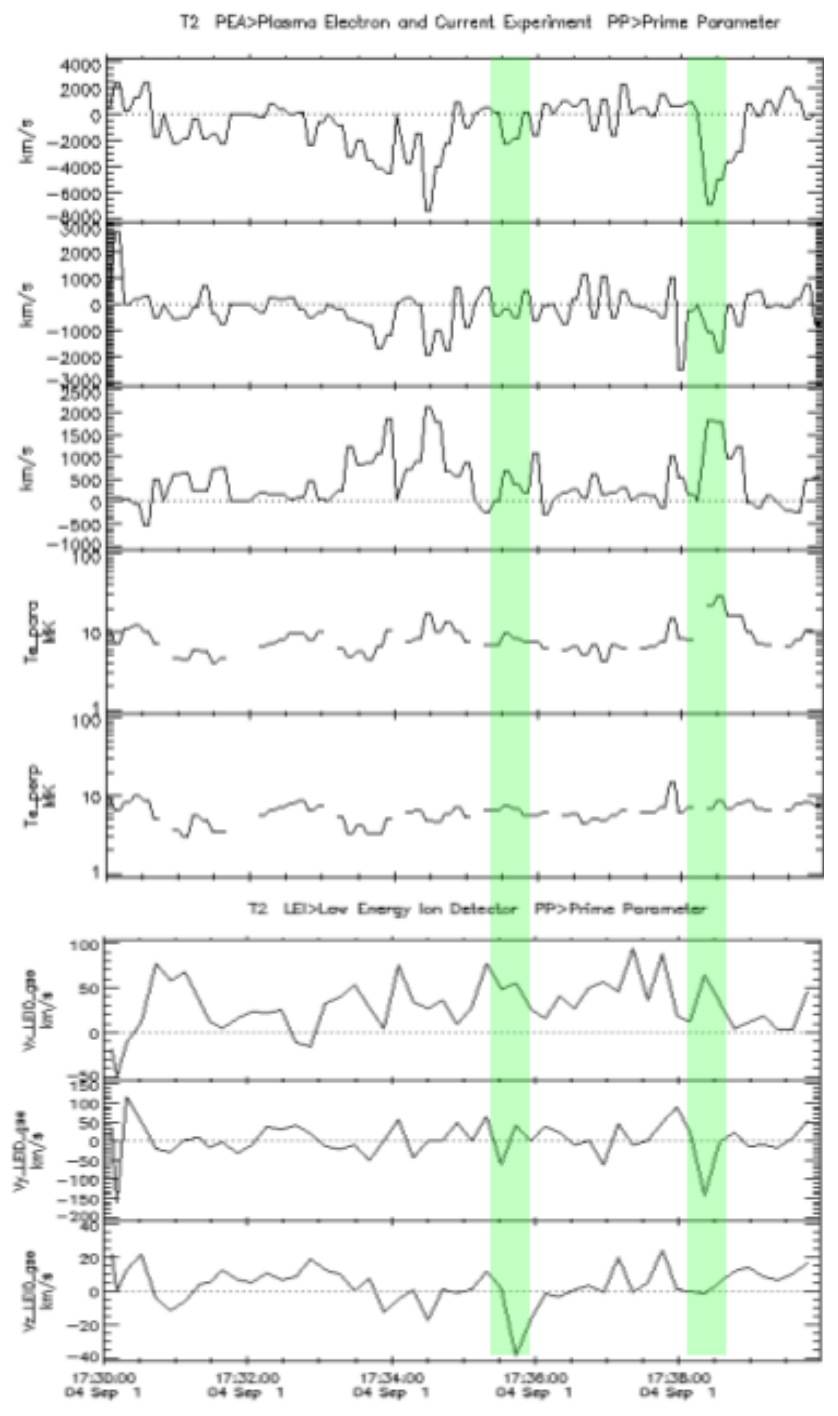

Fig. 4. Low-energy electron data recorded by PEACE on 1 September 2004 are, from top to bottom/top panel: electron velocity, $X$ component $\left(V_{X}\right)$, Y-component $\left(V_{Y}\right)$, Z-component $\left(V_{Z}\right)$, the parallel electron temperature ( $\left.T_{\text {para }}\right)$, and the perpendicular electron temperature $\left(T_{\text {perp }}\right)$. The bottom panel shows, from top to bottom, ion velocity X-component $\left(V_{X}\right)$, Y-component $\left(V_{Y}\right)$, and Z-component $\left(V_{Z}\right)$ recorded by LEID. The time intervals of the frames under study (Fig. 1) are shaded in green.

In the PEACE data, nearly field-aligned, high speed, electron beams $\left(V_{x}=-2300 \mathrm{~km} / \mathrm{s}\right.$ and $\left.V_{x}=-7000 \mathrm{~km} / \mathrm{s}\right)$ were observed at the times of both the selected frames (Fig. 1) There was no obvious event in the corresponding LEID data (see Fig. 4, green shading). During the concerned periods, the parallel electron temperature was higher than the perpendicular electron temperature. The omni-directional flux of (49.2$125 \mathrm{keV}$ ) electrons recorded by NUADU in Energy Channel 3 is compared in Fig. 5 with the corresponding high energy electron flux $(0.2-0.4 \mathrm{MeV})$ recorded by HEED. There is no significant correlation between these records and it can, thereby, be deduced that the up-flowing electron beams with energies $<100 \mathrm{keV}$, spiralling around the field lines to form

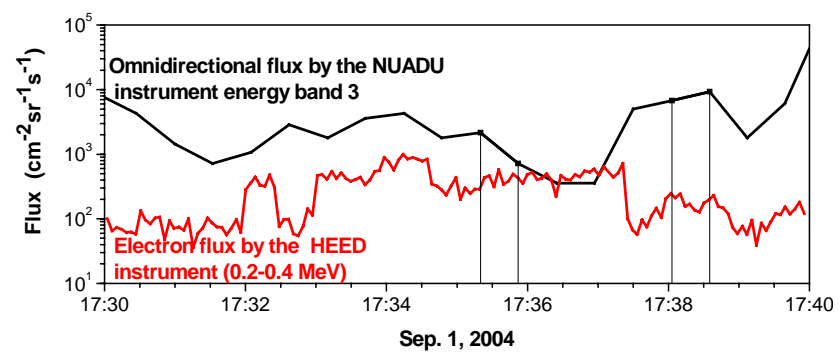

Fig. 5. The omnidirectional flux of electrons (black line) observed by the NUADU instrument in Energy Channel 3 (49.2-125 keV), and the high energy $(0.2-0.4 \mathrm{MeV})$ omnidirectional electron flux (red line) simultaneously observed by the HEED instrument, on 1 September 2004. The vertical black lines indicate the periods of the frames under study (Fig. 1).

ring-like particle distributions, are not influenced by simultaneously up-flowing, high energy, electrons.

\section{The evolution of a dumbbell-type PAD}

On 4 September 2004 from 13:55:14 UT to 13:56:19 UT, the NUADU instrument recorded a sequence of 5 frames in Energy Channel 3, in the course of which particles flowed along, rather than being distributed around, a magnetic field line (see Fig. 6, left side which shows the time sequence from top to bottom). The corresponding PAD plots are displayed on the right side of Fig. 6. Each frame in Fig. 6 was integrated over 4 spins, and the accumulation time for each pixel was $0.125 \mathrm{~s}$. In contrast to what is seen in Fig. 1, there is no trace of a normal PAD background in these data.

Figure 7 shows the location of the TC-2 satellite at the time the frames displayed in Fig. 6 were recorded. During the more than one-minute time interval concerned, a negative magnetic pulse with an amplitude of about 7 nT (Fig. 8) was recorded in ambient magnetic field data. It evolved such that, at 13:55:18 UT, the Y-component of the magnetic field increased by $20 \mathrm{nT}$. Then, at 13:55:22 UT, the X-component of the field decreased by $5 \mathrm{nT}$. Finally, at 13:55:26 UT, the $\mathrm{Z}$-component also decreased by $17 \mathrm{nT}$. The change in the Z-component of the magnetic field resembles that of the Ycomponents, but after an 8-s delay.

During the decrease in the magnetic field, the colour of the frame background in the particle data changed from orange to green (central frame) and returned to orange as the magnetic field again increased (middle to bottom frame). The mean particle fluxes associated with the orange and green pixels were, respectively, $3.2 \times 10^{5} \mathrm{~cm}^{-2} \mathrm{sr}^{-1} \mathrm{~s}^{-1}$ and $1.8 \times 10^{5} \mathrm{~cm}^{-2} \mathrm{sr}^{-1} \mathrm{~s}^{-1}$. The loss cone areas that appear in red (mean flux per pixel $6.4 \times 10^{5} \mathrm{~cm}^{-2} \mathrm{sr}^{-1} \mathrm{~s}^{-1}$ ), initially narrowed towards the field line and, thereafter, were restored. This implies the behaviour of charged particles under circumstances where the first adiabatic invariant $\left(\mu=E_{\perp} / B\right)$ was conserved. 

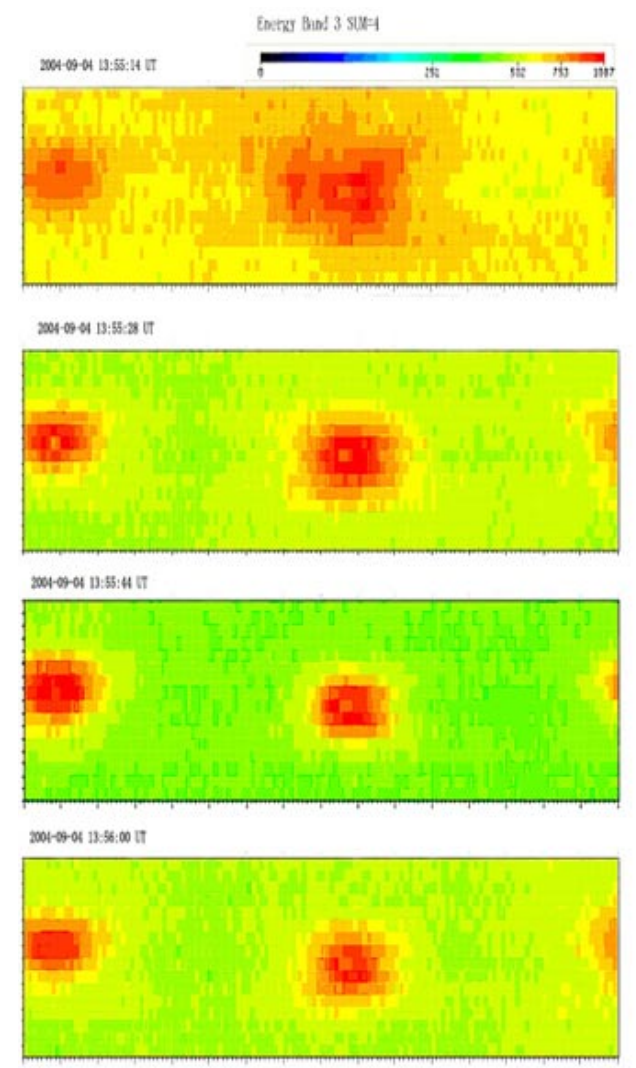

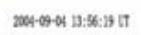

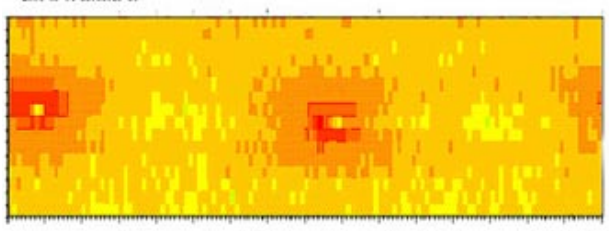

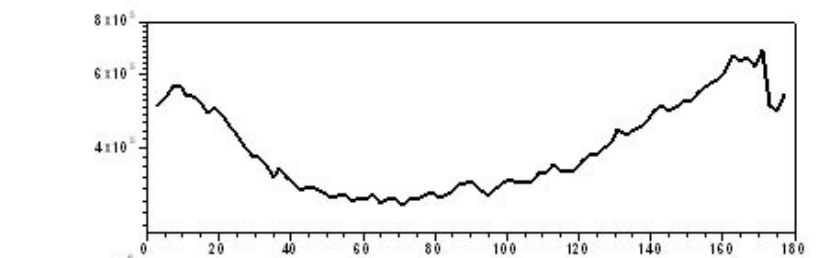

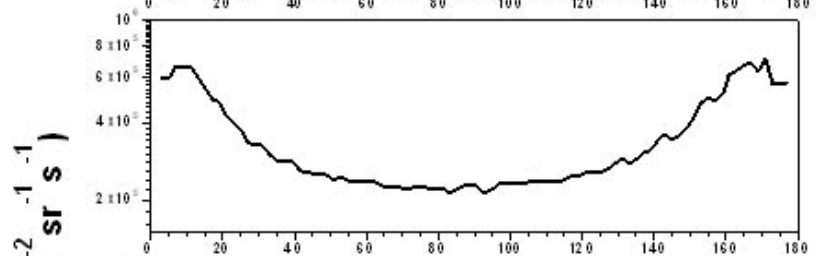

言
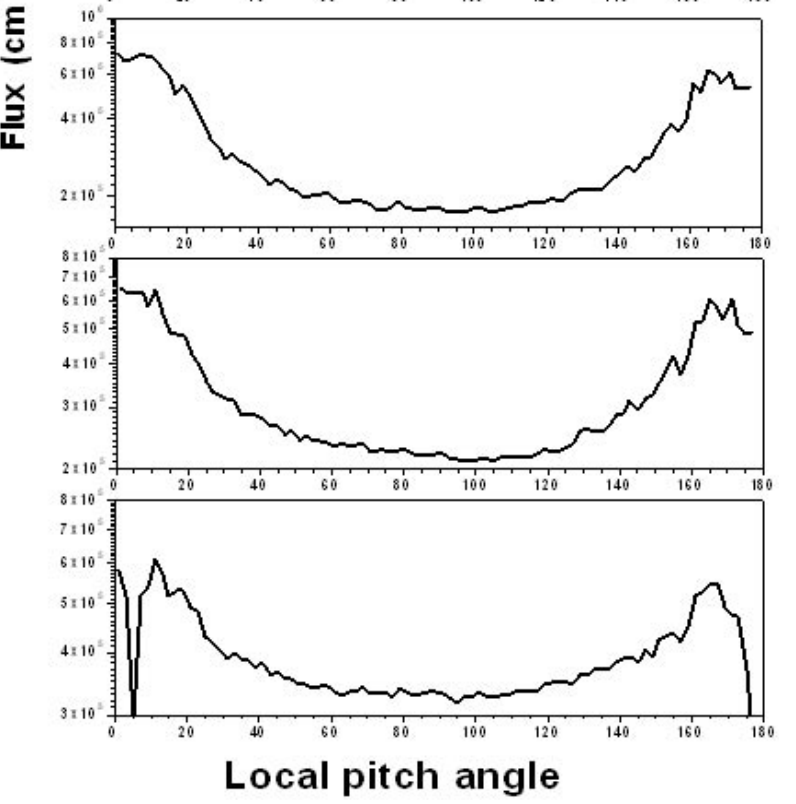

Fig. 6. NUADU data frames (left side) and local PAD plots (right side) for the interval 13:55:14 UT to 13:56:19 UT on 4 September 2004. The data show, from top to bottom, the evolution of dumbbell-type PADs, where each abscissa (left side) covers 128 azimuths, with $2.8125^{\circ}$ to each pixel, and each ordinate (left side) covers 16 elevations, with $11.25^{\circ}$ to each pixel. The colour scales on the top illustrate the particle counts in each pixel. The flux peaks in each local PAD plot (right panels) correspond to the red areas in the frames on the left.

Electrons flowing upwards along the magnetic field line were recorded by the PEACE instrument (Fig. 9). At 13:55:18 UT, the Y-component of the electron beam experienced a sudden injection of particles having velocity $V_{Y}=-1482 \mathrm{~km} / \mathrm{s}$. Thereafter, three further high velocity electron beam enhancements occurred (at 13:55:26 UT, $\boldsymbol{V}_{x}=-4307 \mathrm{~km} / \mathrm{s}$; at $13: 55: 42 \mathrm{UT}, \boldsymbol{V}_{x}=-766 \mathrm{~km} / \mathrm{s}$; and at 13:56:07 UT, $\left.\boldsymbol{V}_{x}=-1232 \mathrm{~km} / \mathrm{s}\right)$. During this active interval, the parallel electron temperature was much higher than the perpendicular electron temperature. The variability of electron fluxes recorded by the HEED instrument (energy range 0.2 to $0.4 \mathrm{MeV}$ ) was consistent with the omnidirectional flux observed by the NUADU instrument in Energy Channel 3 for electrons (49.2-125 keV); see Fig. 10. However, the time resolution of NUADU was $16 \mathrm{~s}$, so that any variability present within this interval would have been smoothed considerably.
The particle observations and comparisons described above indicate that the main component of the dumbbell-type PAD was provided by electron beams covering a rather wide energy region from hundreds of $\mathrm{eV}$ to hundreds of $\mathrm{keV}$.

\section{Discussion and conclusion}

Usually, PADs are observed either with maximum close to $90^{\circ}$, or expanding to the edge of the loss-cone with a pancake-type distribution. The two abnormal PAD events presented above (Fig. 6) are related to up-flowing electron beams. These cases are different than the event described in Sect. 3, concerning a unidirectional electron beam with a normal PAD background, whereas the event described in Sect. 4 concerns a bidirectional electron beam without a normal PAD background. 

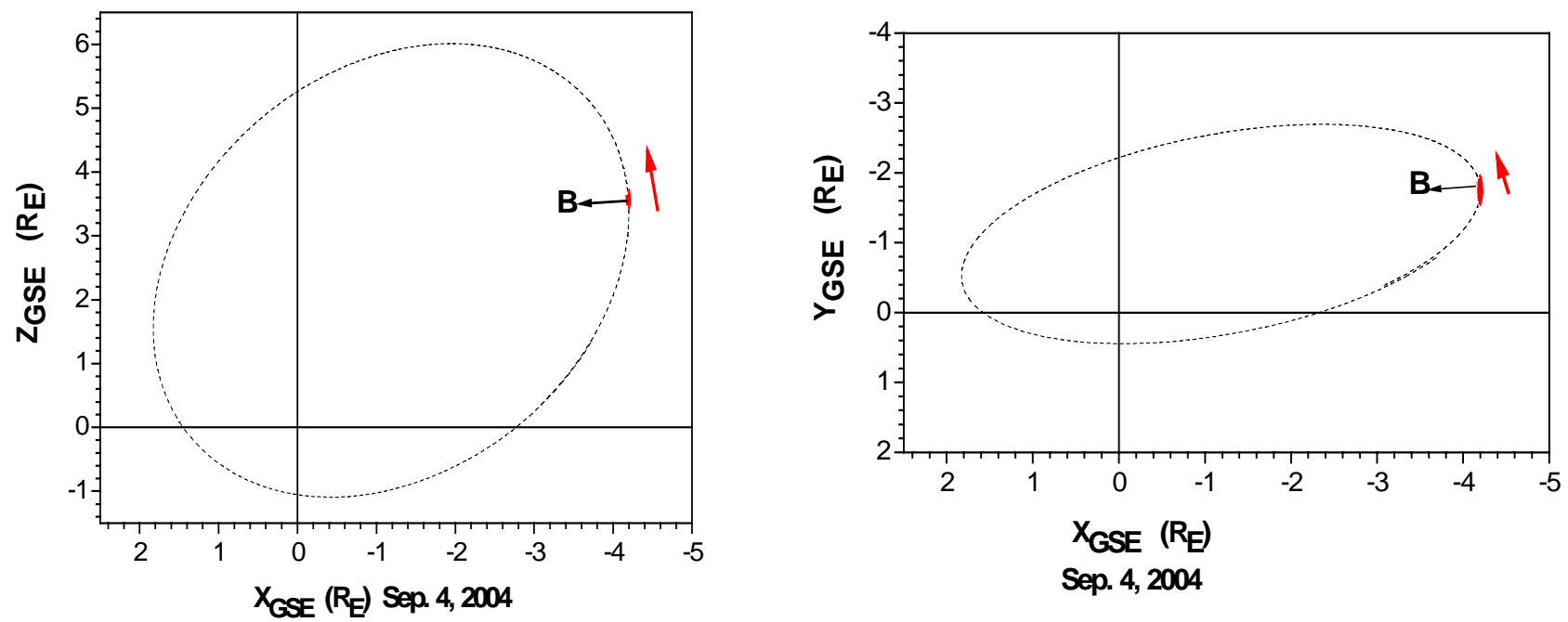

Fig. 7. The location of TC-2 in the Z-X plane (left panel) and in the Y-X plane (right panel) in the GSE system. Dotted lines indicate the orbit of the spacecraft. The red heavy line shows the position of the spacecraft when the frames displayed in Fig. 6 were recorded. Black arrows indicate the magnetic field direction and red arrows the direction of motion of the spacecraft at that time.
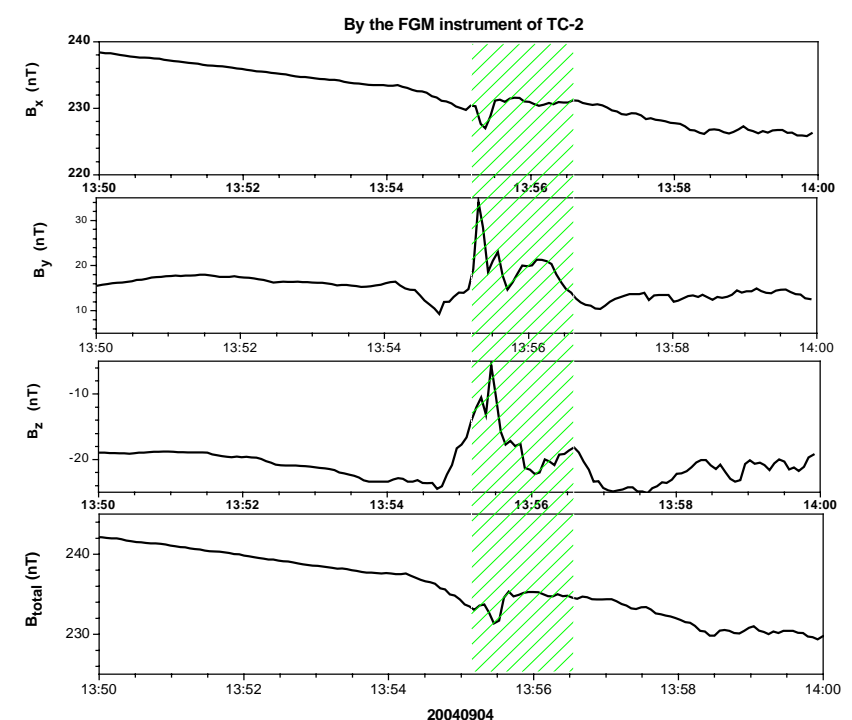

Fig. 8. Magnetic field plots obtained by FRM on 4 September 2004 comprise, from top to bottom, the magnetic field $\mathrm{X}$-component $\left(B_{X}\right)$, the Y-component $\left(B_{Y}\right)$, the Z-component $\left(B_{Z}\right)$, and the total magnetic field intensity $\left(B_{T}\right)$. The time intervals of the frame under study (Fig. 6) is coloured in green.

The magnetic field intensity showed no changes during the event described in Sect. 3, but only a small distortion of the magnetic flux tube. During the evolution (over one minute) of the event described in Sect. 4, a negative magnetic pulse with an amplitude of about $7 \mathrm{nT}$ propagated towards the Earth. PEACE recorded only up-flowing electron beams at low energies at this time but the particle images observed by NUADU indicate the presence of bidirectional electron beams flowing symmetrically along the magnetic field lines.

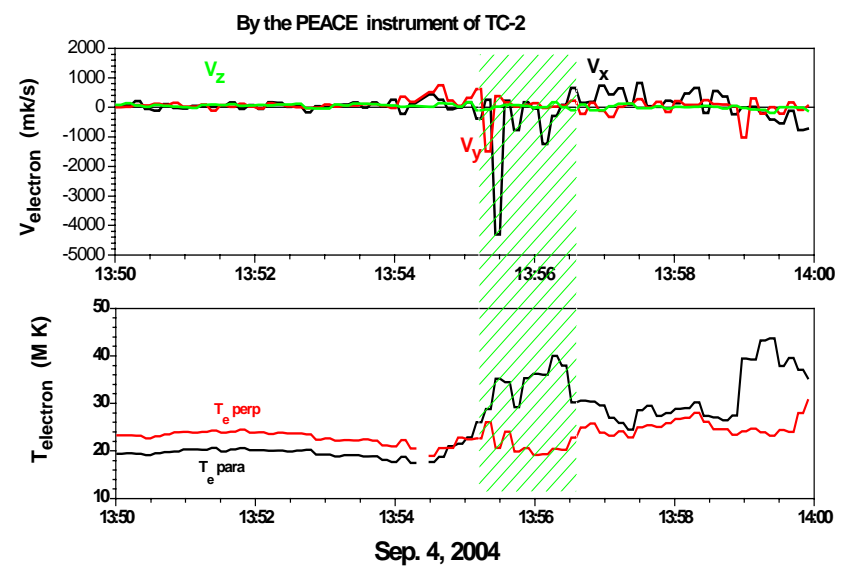

Fig. 9. Low-energy electron velocity and temperature data recorded by the PEACE instrument aboard TC-2 on 4 September 2004. The top panel shows three components of the electron velocity, where the X-component is in black, the Y-component in red, and the Zcomponent in green. The bottom panel shows the parallel electron temperature (black), and the perpendicular electron temperature (red). The period covered by the data under study (Fig. 6) is shaded in green.

The area of the electron PAD of the up-flowing (red pixels in the central part of Fig. 6) at first narrowed in the direction of the field line, and then was partly restored. This implies that there must have been a magnetic mirror point (labelled MP in Fig. 11) not far from the location sampled, which temporarily separated the original magnetic bottle into two parts (regions I and II in the same figure). The temporary magnetic mirror point formed could reflect up-flowing electron beams. Also, it could prevent particles with large pitch angles from entering region II from region I, with the 


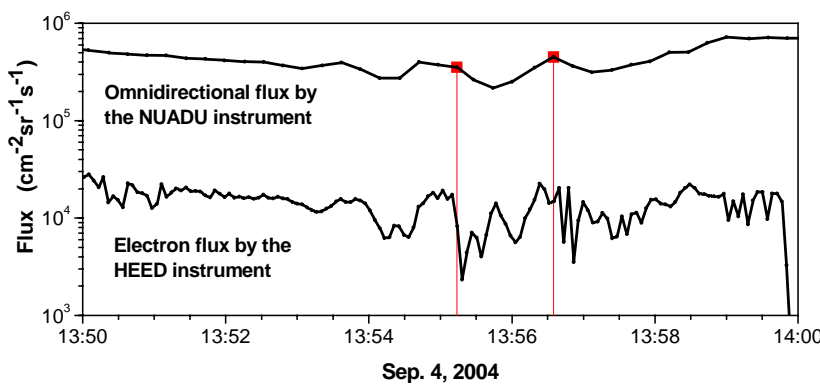

Fig. 10. The omnidirectional electron flux (top line) recorded by NUADU in Energy Channel $3(49.2-125 \mathrm{keV})$ on 4 September 2004 , and the corresponding high energy $(0.2-0.4 \mathrm{MeV})$, omnidirectional, electron flux (bottom line) observed by the HEED instrument. The vertical red lines indicate the period of the observations under study (Fig. 6).

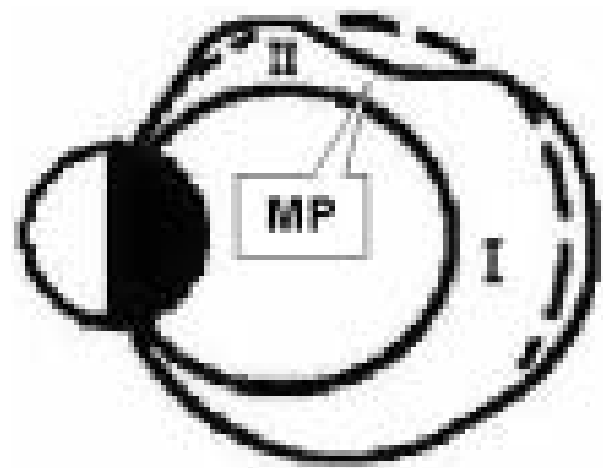

Fig. 11. Schematic illustrating nested magnetic bottles, where the new mirror point (MP) in the magnetic field produced due to the propagation earthward of a magnetic pulse, separates the dipole magnetic field into two regions (labelled I and II).

result that the PAD maps imaged by NUADU did not show a normal PAD background in region II. If we assume that the temporary magnetic mirror point moved earthward, due to the magnetic pulse that propagated along the magnetic field line, so that the reflection distance of the $\mathrm{keV}$ electron beam was reduced, this process would, in effect, constitute a magnetic pump to accelerate these electrons by the Fermi acceleration mechanism. The central red area in the PADs shown in Fig. 6, which are concentrated in the direction of the field line, may indeed comprise evidence of such electron acceleration.

To summarize the account of abnormal PAD events provided above, it was found that a magnetic field line could be easily deformed from its typical dipolar configuration near the HMLBL during geomagnetic activity. When magnetic flux tube variations were transient, so that the original total magnetic field intensity was restored, a pancake-type PAD background was present in the PAD particle map. When the disturbance was strong enough to cause the total magnetic field intensity to show a sustained change, the single magnetic bottle of a dipole magnetic field developed to form a nested pair. In this case, no pancake-type PAD background was observed in the PAD particle map. Further examples of these effects should now be studied.

The formation of a nested magnetic bottle is important with regard to potential particle acceleration, and the viewing capability of the NUADU instrument offers the opportunity to investigate further cases where this configuration is present.

Acknowledgements. This study was supported by the Chinese National Natural Science Foundation Committee grant 40390153, 40125012, and VEGA grant agency project 4064. S. McKennaLawlor acknowledges with appreciation the support of Enterprise Ireland.

Topical Editor T. Pulkkinen thanks T. A. Fritz and another referee for their help in evaluating this paper.

\section{References}

Asnes, A., Friedel, R. W. H., Stadsnes, J., Thomsen, M., Østgaard, N., and Cayton, T.: Statistical pitch angle properties of substorm injected electron clouds and their relation to dawnside energetic electron precipitation, J. Geophys.Res., 110, A05207, doi:10.1029/2004JA010838, 2005.

Boscher, D., Bourdarie, S., Thorne, R. M., and Abel, B.: Influence of the wave characteristics on the electron radiation belt distribution, Adv. Space Res., 26, 163-166, 2000.

Friedel, R. H. W., Reeves, G. D., and Obara, T.: Relativistic electron dynamics in the inner magnetosphere - a review, J. Atmos. Solar Terr. Phys. 64, 265-282, 2002.

Fujimoto, M. and Nishida, A.: Energization and anisotropization of energetic electrons in the Earth's radiation belt by the recirculation process, J. Geophys. Res., 95, 4265-4270, 1990.

Green, J. C and Kivelson, M. G.: Relativistic electrons in the outer radiation belt: differentiating between acceleration mechanisms, J. Geophys. Res., 109, A03213, doi:10.1029/2003JA010153, 2004.

Liu, W. W., Rostoker, G., and Baker, D. N.: Internal acceleration of relativistic electrons by large-amplitude ULF pulsations, J. Geophys. Res., 104 , 17 391-17 407, 1999.

McKenna-Lawlor, S., Balaz, J., Barabash, S., Johnsson, K., Lu, L., Shen, C., Shi, J. Q., Zong, Q. G., Kudela, K., Fu, S. Y., Roelof, E. C., Brandt, P. C., and Dandouras, I.: The energetic NeUtral Atom Detector Unit (NUADU) for China's Double Star Mission and its calibration, Nuclear Inst. \& Methods, A(503) 311-322, 2004.

Williams, D. J.: Energetic ion beams at the edge of the plasma sheet: ISEE 1 observations plus a simple explanatory model, J. Geophys. Res., 86, A7, 5507-5518, 1981. 